\title{
M87 Supermassive Black Hole Review
}

\author{
Author: Mekhala Ganguly \\ Address: 17, Panpara First Lane,Kolkata, West Bengal \\ Affiliation: Bs Ms $2^{\text {nd }}$ year IISER Berhampur \\ Contact no: 9078598357,9831188224; Email id: mekhala19@iiserbpr.ac.in, \\ piyaganguly67@gmail.com \\ University: IISER Berhampur; Govt. ITI Building Engg. School Junction, \\ Brahmapur, Odisha 760010 \\ ORCID: https://orcid.org/0000-0002-1130-9519
}

\begin{abstract}
M87 is a giant elliptical galaxy in the Virgo cluster of galaxies. The radio source has a core which coincides with the nucleus of the galaxy, and a jet of emission which is detected from radio to X-ray bands. A supermassive black hole is assumed to be at the centre of M87 which sends out relativistic particles in the form jets along its axis of rotation. Relativistic particles accelerated in a magnetic field, give out synchrotron radiation. The centre is surrounded by an accretion disc, which is the closest that we can probe into a black hole. High resolution observations are needed to examine the nature of the radio emission closest to the centre of M87. An array of millimetre-band telescopes across the globe were used as an interferometer, called the Event Horizon Telescope, (EHT) to probe the nuclear region. The angular resolution of this interferometer array is 25 microarc sec, at a wavelength of $1.3 \mathrm{~mm}$ and the data was carefully calibrated and imaged. The resulting image shows an asymmetric ring which is consistent with the predictions of strong gravitational lensing of synchrotron emission from hot plasma near the event horizon. In this paper, we review the results of the observations of the radio galaxy, M87, using the Event Horizon Telescope.
\end{abstract}

Keywords: black hole, supermassive black hole, black hole physics, M87 galaxies, gravitation, Event Horizon, telescope, observational astronomy,

\section{Introduction}

The concept of the supermassive black holes came in light with the general relativity theory prediction. We can define the black hole as a massive hole in space-time, with a high gravitational force, from which even light cannot escape.

Being a popular concept of Astrophysics, the black hole has only one-way entry and there is no possible known exit. It is believed that the black holes accumulate millions to billions of solar masses and are present in the centre of each galaxy. 
The gravitational-wave measurements, as well as X-rays, provide immense evidence for the existence of black holes that are made up with the stellar mass. The brightest central part of the black holes is called the Active galactic nuclei (AGNs) (Akiyama et al., 2019b). These AGNs are known to exceed the brightness of the host galaxy's stellar population.

\section{M87 Galaxy}

The M87 galaxy was discovered in the year 1781 by famous scientist Charles Messier. The proximity of this galaxy from the earth is 54 million light-years, being present in the neighboring galaxy of Virgo.

Trillions of stars vastly spread, cluster of globular stars that are approximately 15,000 in number and the massive black hole, collectively forms the M87(Akiyama et al., 2019a), which is an elliptical galaxy. Being immensely bigger in size, the M87 dominates over its neighbouring 2000 galaxies in the Virgo cluster of galaxies.

The size of the M87 galaxy is bigger than the Milky Way galaxy and the diameter of the M87 galaxy is calculated as 240,000 light-years. The magnitude of the M87 galaxy is 9.6 (Akiyama et al., 2019a) which enables this galaxy to be easily seen with the help of a small telescope.

The month of May is considered as best to observe this galaxy with the help of a telescope. The visible, as well as the infrared light, are used to produce the Hubble image of M87.

A stream of subatomic particles is formed due to the release of the energy bypassing of gaseous material from the centre of the galaxy to the black hole. It results in generating ultrarelativistic particles which give out synchrotron radiation in every part of the electromagnetic wave.

The centre of the Virgo galaxy is being occupied by the M87 galaxy because of its domination and the gravitational pull, sidelining the small galaxies, which are without the cluster now, due to the dominance of the M87 galaxy. The stars of the M87 galaxy occupy a spheric symmetric shape (Akiyama et al., 2019b).

The whole M87 galaxy has an elliptical shape, quite different from the other galaxies that are usually spherical in shape. The M87 galaxy is void of the dust lanes and seems featureless with the luminosity at the centre when seen from the far.

With the increase in the distance from the core of the M87 galaxy, the density of population goes on decreasing. Being the strongest source of multi-wavelength radiation, mainly radio waves, the galactic envelope of the M87 exceeds a radius of 490,000 light-years. 
The relativistic jet is made up of the matter that is ejaculated from the massive black hole and it exceeds up to five thousand light-years. At the core, the jet is constrained to $60^{\circ}$, within 0.8 pc (2.6 light-years) of the core, to $7^{\circ}$ at twelve parsecs and about $16^{\circ}$ at two parsecs (M87 | Black Hole, Distance, \& Facts | Britannica, 2019).

We have already said that the emission is synchtrotron radiation caused by the relativistic electrons radiating in the magnetic field. Synchrotron radiation is polarised.

These electrons have an energy of $5.1 \times 10^{\wedge} 56$ ergs. Energy produced by milky way galaxy in one second is $5 \times 10^{\wedge} 36$ joules (Akiyama et al., 2019a). When compared to this energy, it is discovered that the M87 galaxy produces $10^{\wedge} 13$ times more energy than the milky way galaxy.

The pictures M87 were taken in the year 1999 by the Hubble Space Telescope that shows M87's jet motion to be five to six times the speed of the light. This phenomenon is called the apparent superluminal motion. The interstellar medium of the M87 contains a diffuse gas. This diffuse gas is comprised of elements that are emitted by the evolved stars.

The primary component of the active galactic nucleus is the giant black hole that is present in the core of the M87 galaxy. The pictures of this black hole were captured with the Event Horizon Telescope in the year 2017 and further on 10 April 2019, the full processed images were released again of the same.

\section{M87 Black Hole at the Centre}

The core of the M87 galaxy consists of a supermassive black hole. The black hole accumulates the centre of the galaxy.

It is estimated that the mass of the black hole of the M87 galaxy is billions of times more than the Sun and Earth. According to the estimates, it ranges from $(3.5 \pm 0.8) \times 10^{\wedge} 9 \mathrm{M}$ to $(6.6 \pm 0.4) \times 10^{\wedge} 9 \mathrm{M}$, surpassed by $7.22+0.34-0.40 \times 10^{\wedge} 9 \mathrm{M} \odot$ in 2016. (M87 | Black Hole, Distance, \& Facts | Britannica, 2019)

The measurements of the black hole mass were released by the Event Horizon Telescope in April 2019. This mass of the black hole is the highest mass which is calculated for such an object to date.

The black hole is being surrounded by the rotating disk that is made up of ionized gas. This disk is perpendicular to the relativistic jet. The velocity with which the disk rotates is 1000 $\mathrm{km} / \mathrm{s}$ and it longs for a span, with a maximum diameter of $0.12 \mathrm{pc}(25,000 \mathrm{AU} ; 0.39$ ly; 3.7 trillion km) (Akiyama et al., 2019a). 
In comparison, from the sun, the Pluto averages $39 \mathrm{AU}$. The estimated rate with which the gas accreted onto the black hole is one solar mass every ten years (M87 | Black Hole, Distance, \& Facts | Britannica, 2019).

The black hole radius of the M87 galaxy is being calculated as $5.9 \times 10-4$ parsecs. This radius is 120 times the distance between the Sun and the earth, which is 120AU.

\section{Interferometry}

This is the technique used for the extraction of information with the help of electromagnetic waves. The electromagnetic waves cause interference due to their superimposition and results in the releasing of the information (LIGO Lab | Caltech, 2019).

It works in the principles of Michelson's interferometer where two beams from the same source are combined to give interference pattern. The amplitude and phases of these fringes are measured as the source is tracked in the sky.

These amplitudes are the Fourier transform of the brightness distribution of the sky. Fast Fourier algorithm is used to transform these amplitude and phases to the sky brightnes distribution. Further image processing is carried out clean the image of certain artefacts of the image processing.

\section{Very Long Baseline Interferometry}

This is called as the VLBI, which is used in radio astronomy and is a type of astronomical interferometry. In the case of VLBI, the astronomical radio source sends the signal which is called quasar, that is then collected by the multiple radio telescope present on the Earth or in space(LIGO Lab | Caltech, 2019).

The Very Long Baseline Interferometry is considered to be the most powerful technique for radio astronomy. With the help of VLBI, one can have the details of the universe with much clarity, better than before.

This is possible because of the linking of the radio telescopes that are widely separated (LIGO Lab | Caltech, 2019). To see the black holes, as well as the surface of the stars, it becomes very easy with very long baseline interferometry extending over thousands of kilometres across various countries all over the world.

With this VLBI, it has become easy to keep a track of the drift of the continents, all from the home. 


\section{EHT}

The Event Horizon telescope project, which has been in the process of development and use for around two decades, links up eight radio telescopes in Arizona, Spain, Mexico, Antarctica and other places around the world to form a virtual instrument the size of Earth. The Event Horizon Telescope creates the image of a black hole shadow owing to the precise coordination of a worldwide telescope network (Akiyama et al., 2019a).

To enhance the capability of the Very Long Baseline Interferometry, the international collaboration is formed to make the improvement a long-term process. To form an Earthsized interferometer so that the radio dishes can be linked across the globe, the EHT has been used (Akiyama et al., 2019b).

The emission region size of the two supermassive black holes is being measured with the EHT. One black hole is present at the centre of the Milky Way Galaxy while the other one is present in the centre of the Virgo A Galaxy.

The size gets a match in both cases of the silhouette that has predicted and which was formed by the black hole's extreme lensing light. The black hole accretion can be imaged and features of dynamic evolution can be taken because of the addition of the key millimeter and submillimetre wavelength specifically on the high altitudes.

The EHT project is comprised of theoretical studies as well as simulation studies. The questions which are being framed from the study on the boundary of the black hole may be answered effectively very soon with the help of observations (Akiyama et al., 2019a).

There is a need for investment on the global level for creating a fundamentally new instrument by linking the EHT as well as the telescopes which use the novel system (Akiyama et al., 2019b). The instruments need the angular resolving power, which has the most possibility even from the earth's surface.

The EHT team is focused to get the black hole information more effectively with the use of increased sensitivity as well as the resolving power, which would be done in the coming years.

\section{Results}

To check the observations of 2009-2013 about the signal-to-noise ratio and U.V. coverage in being sufficient with respect to the simple asymmetric ring models along with constraining source geometric properties, the tests are being designed by utilizing synthetic VLBI observations. 
The imaging software helps to generate the synthetic observations with the help of four emission models of sampling. GRMHD1, GRMHD2, MODEL1, MODEL2 are the four sampling models with which the synthetic observations are done (Wielgus et al., 2020). These models are generated by covering the thermal error budget according to past observations.

In the synthetic observations, the station-based gain errors are being incorporated with the corruption from time-dependent. To know the model-related bias as well as for assessing to what extent the geometric parameters are being covered with the simplified models and the one done with the EHT.

The data under fitting of the sample set with respect to the array result of 2017, is noted for the parameter posteriors of the artificial narrowing (the complex GC model is considered for capturing the full posterior n EHTC). The thermal error budget and the data is generally much closer with ML estimators for 2009-2013(Wielgus et al., 2020).

The number of effective degrees of freedom is being complicated for the estimation because the small array is considered as per the station gains of the time-dependent station (on the same baselines, 2-3 telescopes were used for observation with missing detections in 20092013, at the same time) (Akiyama et al., 2019f).

It may happen that the number of data points is being smaller than the number of model parameters, formally. As a result, the EHTC IV and the EHTC VI process cannot be repeated, and goodness-of-fit statistic cannot be used here.

\section{Discussion}

The several nights of five years 2009, 2011, 2012, 2013, and 2017 were covered for the observation with the five different $1.3 \mathrm{~mm}$ VLBI. The data set for the whole year is being used while fitting the data with single data which is availed by the several days.

In the EHT images of 2017, the north-south asymmetry is along with all other sets of data. It was notified that the variation in the position angle in some GRMHD stimulation (Akiyama et al., 2019e).

So, on the simulation parameters, the observations provide the new constraints over the source variability. That includes the electron heating model, black hole spin, and accretion flow magnetization. 
In interpreting this variability, the accretion flow spin of the accretion disk of the black hole may be important as expected with the unmodelled physical effects. Various other constraints are taken into account to determine the morphology of the disk.

The order is $\sim 15 \mathrm{M}$ as per the observation of 2017 to $\sim 7900 \mathrm{M}$ between the campaigns of 2009 and 2017.

The correlation timescale of M87 is least expected than a few tens of M. The turbulent accretion flow association from the transient features makes it critical of a longer span for the decoupling stable (Akiyama et al., 2019f).

It must be allowed to discriminate between the GRMHD model variables as per the estimated position angle. This provides physical parameters constraint of $\mathrm{M} 87^{*}$ as well as for quantitative time-domain studies, opening an exciting new avenue.

In a collaborated framework, we provide the independent observations of M87* that are 5 in number. And the stability, as well as source morphology, get important insight.

These data which were gathered as a result of the visibility observations from the ground taken on these various days were in the form of Fourier transformation (Akiyama et al., 2019b)with the maximum magnetic flux density being at the zeroth component as the energy is maximum at the centre(Akiyama et al., 2019c).

To estimate closure, bi-spectral averaging is used and the visibility amplitudes are estimated with incoherent averaging on each scan. All simultaneous visibility amplitudes were incoherent when both parallel hand polarization was available.

The two $480 \mathrm{MHz}$ bands were contained in the 2009-2013 setup of frequency, which was centred at 229.089 as well as $229.601 \mathrm{GHz}$. All simultaneous visibility amplitudes were incoherently averaged whenever there was the availability of both parallel hand polarization components.

The baseline length is given of the different projects for non-redundant baseline along with the number of detections and summarization of the data sets(Wielgus et al., 2020).

The same visibility is yielded as independent observations of redundant baselines. The availability of non-redundant CPs with the number is being indicated. We cannot get the absolute visibility phases in observations of non-phases referenced VLSI. 
It is observed from the collected data and observations that there can be various angular emissions with very small differences and hence the model was based on the resultant of the angular emission GRMHD m (Akiyama et al., 2019d).

Phase and amplitude are calculated from the observations at this wavelength of $1.3 \mathrm{~mm}$. Amplitude can be easily calculated but if the phase is missed, we cannot understand the orientation of the two points even though the number of points is determined by the observational data (Akiyama et al., 2019d).

While gathering data using a wavelength of $1.3 \mathrm{~mm}$, the atmospheric conditions play a major role and can cause hindrances resulting in errors unlike the observations taken at a larger wavelength like $6 \mathrm{~cm}$ or more (Akiyama et al., 2019b).

\section{Conclusion}

The asymmetric ring model is being used, which is being motivated by the EHT imaging and modeling. For the observations of the M87 geometric modeling of the year, 2009-2017 has been performed.

It is being observed that the fitting ring diameter is being stable. The relevance of the shadow of the supermassive black hole with the ring diameter is strongly favored.

The intrinsic variability is being in its position angle as well as in the total flux density. In the previous 2013 observation that is unpublished, the east-west direction has the brightness asymmetry.

In comparison to the 2017 observations, the 2009-2013 data sets are comparatively weaker. The modest structural variability is shown along with the persistent ring structure. For the M87* model, variability and persistence offer important constraints.

\section{Future questions}

In the science of space, precisely astronomy, there is no end for the excitement when we get to solve various mysteries and longstanding theories based on the first observational or experimental results. This is one of the reasons why the universe and the various astronomical objects never fail to amuse the astronomers all over the world.

Imaging the M87 blackhole for the first time through Event Horizon Telescope, we analysed the observations and received answers to many of our questions. However, some questions still remain unanswered. 
The first among them is How are the enormous jets of hot, fast matter produced by these supermassive blackholes? Do they vary in length? If so, what factors cause these differences?

It is known to us that all supermassive black holes absorb most of the nearby matter past their event horizons by chewing or swallowing them up and eject the residual out into universe at a speed which is almost same as that of the speed of light in blazing towers, which astrophysicists call "relativistic jets."

Moreover, the M87 black hole which is at the centre of Virgo A (also called Messier 87) is notorious for its impressive relativistic jets, spewing matter and radiation all over space. Its relativistic jets are enormously huge, approximately of 500 light years and can fully escape the surrounding galaxy.

Another question lies here is that Can we relate general relativity and quantum mechanics together while explaining the black hole phenomenon and its morphology?

For a long while, we have worked using two different sets of rules: General relativity by Einstein, which covers very big things like gravity, and quantum mechanics, which deals with very small things. However, the problem is that, those two theories directly contradict one another: Quantum mechanics can't explain gravity, and relativity can't explain quantum behaviour.

The objective is to compare these observational results with theoretical studies which form the grand unified theories combining theory of gravity and quantum mechanics. 


\section{References:}

- Akiyama, K., Alberdi, A., Alef, W., Asada, K., Azulay, R., Baczko, A.-K., Ball, D., Baloković, M., Barrett, J., Bintley, D., Blackburn, L., Boland, W., Bouman, K.L., Bower, G.C., Bremer, M., Brinkerink, C.D., Brissenden, R., Britzen, S., Broderick, A.E. and Broguiere, D. (2019a). First M87 Event Horizon Telescope Results. I. The Shadow of the Supermassive Black Hole. The Astrophysical Journal, [online] 875(1), p.L1. Available at: https://pureportal.spbu.ru/en/publications/first-m87-event-horizontelescope-results-i-the-shadow-of-the-sup.

- Akiyama, K., Alberdi, A., Alef, W., Asada, K., Azulay, R., Baczko, A.-K., Ball, D., Baloković, M., Barrett, J., Bintley, D., Blackburn, L., Boland, W., Bouman, K.L., Bower, G.C., Bremer, M., Brinkerink, C.D., Brissenden, R., Britzen, S., Broderick, A.E. and Broguiere, D. (2019b). First M87 Event Horizon Telescope Results. II. Array and Instrumentation. The Astrophysical Journal, 875(1), p.L2.

- Akiyama, K., Alberdi, A., Alef, W., Asada, K., Azulay, R., Baczko, A.-K., Ball, D., Baloković, M., Barrett, J., Bintley, D., Blackburn, L., Boland, W., Bouman, K.L., Bower, G.C., Bremer, M., Brinkerink, C.D., Brissenden, R., Britzen, S., Broderick, A.E. and Broguiere, D. (2019c). First M87 Event Horizon Telescope Results. III. Data Processing and Calibration. The Astrophysical Journal, [online] 875(1), p.L3. Available at: https://ui.adsabs.harvard.edu/abs/2019ApJ...875L...3E/abstract.

- Akiyama, K., Alberdi, A., Alef, W., Asada, K., Azulay, R., Baczko, A.-K., Ball, D., Baloković, M., Barrett, J., Bintley, D., Blackburn, L., Boland, W., Bouman, K.L., Bower, G.C., Bremer, M., Brinkerink, C.D., Brissenden, R., Britzen, S., Broderick, A.E. and Broguiere, D. (2019d). First M87 Event Horizon Telescope Results. IV. Imaging the Central Supermassive Black Hole. The Astrophysical Journal, 875(1), p.L4.

- Akiyama, K., Alberdi, A., Alef, W., Asada, K., Azulay, R., Baczko, A.-K., Ball, D., Baloković, M., Barrett, J., Bintley, D., Blackburn, L., Boland, W., Bouman, K.L., Bower, G.C., Bremer, M., Brinkerink, C.D., Brissenden, R., Britzen, S., Broderick, A.E. and Broguiere, D. (2019e). First M87 Event Horizon Telescope Results. V. Physical Origin of the Asymmetric Ring. The Astrophysical Journal, [online] 875(1), p.L5. Available at: https://iopscience.iop.org/article/10.3847/2041-8213/ab0f43.

- Akiyama, K., Alberdi, A., Alef, W., Asada, K., Azulay, R., Baczko, A.-K., Ball, D., Baloković, M., Barrett, J., Bintley, D., Blackburn, L., Boland, W., Bouman, K.L., Bower, G.C., Bremer, M., Brinkerink, C.D., Brissenden, R., Britzen, S., Broderick, A.E. and Broguiere, D. (2019f). First M87 Event Horizon Telescope Results. VI. The Shadow and Mass of the Central Black Hole. The Astrophysical Journal, [online] 875(1), p.L6. Available at: https://arxiv.org/abs/1906.11243 [Accessed 9 Oct. 2019].

- LIGO Lab | Caltech. (2019). What is an Interferometer? [online] Available at: https://www.ligo.caltech.edu/page/what-is-interferometer [Accessed 21 Jul. 2019].

- M87 | Black Hole, Distance, \& Facts | Britannica. (2019). In: Encyclopcedia Britannica. [online] Available at: https://www.britannica.com/place/Virgo-A.

- Wielgus, M., Akiyama, K., Blackburn, L., Chan, C., Dexter, J., Doeleman, S.S., Fish, V.L., Issaoun, S., Johnson, M.D., Krichbaum, T.P., Lu, R.-S., Pesce, D.W., Wong, G.N., Bower, G.C., Broderick, A.E., Chael, A., Chatterjee, K., Gammie, C.F., Georgiev, B. and Hada, K. (2020). Monitoring the Morphology of M87* in 20092017 with the Event Horizon Telescope. The Astrophysical Journal, 901(1), p.67. 